
temps de la convergence?

\title{
Éducation aux TIC, cultures informatique et du numérique : quelques repères historiques
}

ICT Education, Computers, and Digital Literacies: A Historical Perspective

Béatrice Drot-Delange et Éric Bruillard

\section{(2) OpenEdition}

Journals

Édition électronique

URL : http://journals.openedition.org/edc/3393

DOI : 10.4000/edc.3393

ISSN : 2101-0366

Éditeur

Université Lille-3

Édition imprimée

Date de publication : 30 juin 2012

Pagination : 69-80

ISBN : 978-2-917562-07-9

ISSN : $1270-6841$

Référence électronique

Béatrice Drot-Delange et Éric Bruillard, «Éducation aux TIC, cultures informatique et du numérique quelques repères historiques », Études de communication [En ligne], 38 | 2012, mis en ligne le 30 juin 2014, consulté le 19 avril 2019. URL : http://journals.openedition.org/edc/3393 ; DOI : 10.4000/ edc.3393

Ce document a été généré automatiquement le 19 avril 2019

(c) Tous droits réservés 


\title{
Éducation aux TIC, cultures informatique et du numérique : quelques repères historiques
}

\author{
ICT Education, Computers, and Digital Literacies: A Historical Perspective
}

\author{
Béatrice Drot-Delange et Éric Bruillard
}

1 La prolifération des objets numériques et l'étendue de leurs utilisations sont telles que des observateurs ou des chercheurs n'hésitent pas à parler de cultures numériques. L'information est au cœur de ces objets, autorisant dès lors à parler de culture informationnelle. Mais elle est aussi au cœur de l'informatique.

2 Si l'éducation aux technologies de l'information et de la communication (TIC) devrait tenir compte des cultures communes et partagées, ou supposées telles, par les élèves, la définir aujourd'hui demande aussi de mieux cerner l'« objet » informatique, en l'ancrant dans une perspective historique, tant dans les cultures qui lui sont associées que dans la manière de penser cet objet. En effet, les évolutions qu'a connues l'informatique pourraient laisser penser que nous sommes passés, en une trentaine d'années, des cultures informatiques focalisées sur le code et la connaissance des langages à des cultures numériques centrées sur les usages, grâce à des interfaces toujours plus conviviales et transparentes. Or, nous montrerons dans une première partie que ces deux types de cultures coexistent aujourd'hui et qu'il ne s'agit pas d'une évolution de l'une vers l'autre. Les discours sur les cultures informatique et du numérique renvoient en fait à une conception de l'informatique qu'il convient de mettre en perspective pour pouvoir réfléchir sur la thématique de l'« éducation à » concernant les TIC. Dans une deuxième partie, nous exposerons trois visions de l'informatique qui nous semblent particulièrement significatives pour éclairer ce débat. 


\section{Cultures informatiques et numériques : des cultures distinctes?}

3 Les cultures renvoient tant à l'étude des valeurs et des normes de groupes (sociaux, générationnels, professionnels, communautaires, etc.) qu'aux disciplines scientifiques, technologiques, littéraires, artistiques, etc. et à la connaissance de leurs productions. Si les cultures informatiques sont étudiées selon ces deux dimensions, il semble que les cultures du numérique caractérisent davantage les usages, par des groupes spécifiques, d'objets informatisés.

\section{Cultures informatiques : une volonté d'émancipation?}

4 Des groupes partageant leur passion de l'informatique, amateurs éclairés ou professionnels, ont fait l'objet de nombreux travaux sociologiques. Ainsi les hackers - au sens originel du terme, passionné d'ordinateur - et leur culture, née dans les années 1970 aux États-Unis, ont particulièrement été étudiés (Turkle, 1984; Breton, 1990). L'expression «culture informatique » serait selon Breton (1990) apparue aux États-Unis au milieu des années 1970 en opposition à une utilisation militaire de l'informatique. Dans un contexte où les progrès concernant les matériels étaient considérables, mais où les grands projets n'avançaient pas, tels ceux de traduction automatique, renvoyer l'informatique à la sphère du culturel évitait de se poser les questions sur ses finalités et ses usages. En France, dans les années 1970-1980, l'expression «culture informatique », selon Proulx (1990), rassemblait plusieurs mouvements de pensées et d'actions prônant la promotion de l'informatique : égalitaristes tels les premiers hackers, militants tels les groupes de pression pour le développement d'une «culture technique», politiques gouvernementales pour la promotion d'une « culture scientifique et technique ». Pour ses militants, la culture technique est jugée nécessaire pour que tout individu puisse agir avec et sur son environnement, gage de sa liberté et de son indépendance. La culture technique se définirait alors par la possession d'un minimum de connaissances et de savoir-faire (De Noblet, 1982). La culture informatique participe de ce mouvement (Arsac, 1987 ; Mirabail, 1990). À la fin des années 1990, il en est de même des communautés des logiciels libres (Demazière, Horn \& Zune, 2009), le plus souvent militantes, dont le développement est basé sur le bénévolat et le volontariat des contributeurs. Ce qui fonde l'existence de ces groupes, c'est la technique informatique et la place qu'elle occupe dans les rapports sociaux de pouvoir : mouvement de résistance à l'informatique d'entreprise, à l'informatique dite propriétaire, etc., mais également formes de reconnaissance personnelle.

5 La promotion de la culture informatique est née de mouvements sociaux visant à émanciper l'individu et à lui donner la maîtrise de son environnement. L'essor de la micro-informatique, avec le tableur notamment, a permis à des « amateurs éclairés » de prendre des positions de pouvoir au sein des entreprises. Cette première vague a concerné plutôt les cadres. Une seconde vague s'est étendue à d'autres personnels, dans l'informatisation de nombreux postes de travail. Elle a été favorisée par le développement des interfaces graphiques et de la manipulation directe, qui se traduit abusivement par le concept marketing d'interfaces conviviales. 
6 Ainsi, en trente ans les objets informatisés se sont multipliés, les utilisations et les utilisateurs aussi. Les cultures informatiques persistent, mais elles cohabitent maintenant avec ce qu'il est convenu de dénommer les cultures du numérique.

\section{Cultures du numérique : l'autonomie des utilisateurs?}

7 Les utilisations des objets informatisés, toujours en croissance, et leurs appropriations par des groupes sont l'objet de recherches en sciences de l'information et de la communication ou en sociologie, qui se font l'écho des cultures multiples du numérique (Casili, 2011; Denouël \& Granjon, 2011). Cet avènement des cultures numériques permettrait à l'informatique, selon Jouët (2011), de gagner d'autres sphères, telles les sphères médiatiques et culturelles, grâce à une moindre technicité nécessaire. Cette simplification, via la micro-informatique puis le développement du web, aurait permis à d'autres personnes que les amateurs d'informatique de s'emparer de ces technologies. Ce serait donc ainsi la démocratisation d'une informatique « sans le savoir » (Jouët, 1990).

Le phénomène le plus récurrent dans la littérature concernant les cultures numériques est celui de la « disparition des adultes » au profit d'un surinvestissement sur la jeunesse (Lobet-Maris, 2011). Cette génération est supposée être particulièrement à l'aise avec les objets informatisés parce que née avec et les utilisant quotidiennement. Cependant, des travaux montrent que cette hypothèse est loin d'être fondée, les compétences techniques développées par les élèves ayant un caractère limité et local, associé à une faible conceptualisation et à une absence de verbalisation des pratiques ordinaires (Baron, Bruillard, 2008 ; Fluckiger, 2008).

9 Postuler l'utilisateur comme compétent, autonome et agissant, c'est oublier sa vulnérabilité (Voirol, 2011). « C'est à renoncer à l'approche classique, axée sur le principe de la formation ou de la 'culture cultivée', qu'invite le paradigme des usages ». La culture apparait alors comme une production ordinaire articulée à des " arts de faire quotidiens » (p.136). C'est ce que révèle aussi l'expression « fracture numérique » qui présuppose des aptitudes d'appropriation chez tous (Granjon, 2011). Perriault (1990) s'appuyant sur les travaux de Greenfield sur les jeux vidéo expliquait déjà, qu'outre les caractéristiques de la pensée induite par les jeux vidéo (multiplicité des points de vue, pensée de type inductif, aptitude à construire un raisonnement formel, etc.), d'autres traits semblaient déterminants pour expliquer la pratique des enfants, notamment l'aptitude à la socialisation: le recours aux parents, amis, magasins, etc. pour résoudre un problème. C'est là une caractéristique de certains milieux sociaux, principalement militants. Dans les études sur les non-usagers d'internet, on retrouve cette idée de passer par d'autres personnes, à travers la notion de proxy (voir par exemple, AWT, 2007). Cependant, cette vision est contrebalancée par la thématique de l'empowerment et de l'accroissement de l'autonomie des utilisateurs des réseaux sociaux, comme en témoigne par exemple le fait que des malades, devenus des experts de leur propre maladie, utilisent les outils de communication pour être acteurs dans la gestion de leur santé (Dupagne, 2011).

10 Les évolutions techniques, notamment la micro-informatique, ont modifié les relations de pouvoir, en élargissant le cercle des amateurs. Pourtant ce n'est pas pour autant que les cultures informatiques ont disparu. Au contraire, la technicité reste présente tant parmi les professionnels de l'informatique que parmi les utilisateurs. Les métiers de l'informatique se sont développés et diversifiés avec l'explosion du web, même si ces professionnels sont encore l'objet de très peu de recherches. Les militants du logiciel libre 
et de l'open source connaissent des succès suffisamment importants pour qu'on assiste aujourd'hui à un phénomène de dissémination de leurs valeurs à d'autres secteurs que celui de l'informatique, tels que les domaines littéraires, artistiques et même politiques. Les artistes du Net Art (Fourmentraux, 2011) mènent des expérimentations autour des langages de programmation, des environnements web, etc. mobilisant ainsi des compétences techniques pointues.

11 Mais postuler la compétence et l'autonomie de l'utilisateur, ce serait oublier la contrainte (Voirol, 2011) : les utilisateurs ne peuvent faire que ce que les machines les autorisent à faire. Peu d'utilisateurs sont en fait capables de contester, de modifier l'environnement préfabriqué qu'on leur propose (Doueihi, 2008). De nombreux utilisateurs sont amenés à programmer, sans avoir nécessairement reçu de formation pour cela. En effet, ils utilisent des applications génériques (traitements de texte, tableurs, retouche d'image, bases de données, sites web, etc.) qu'ils doivent parfois adapter à leurs besoins en développant des routines, en utilisant des fonctions, etc. Ce phénomène, repéré très tôt, qualifié en anglais de end-user programming, est à l'origine associé à l'utilisation du tableur. Ce côté programmation offert à des non-professionnels recèle des risques qui, eux aussi, ont été analysés très tôt (Brown et Gould, 1987). Robert Panko, qui a longtemps analysé les erreurs dans les feuilles de calcul, estime que la proportion contenant des erreurs oscille entre $20 \%$ et $40 \%$, conduisant à une abondante littérature sur l'analyse des erreurs, sur les méthodes d'audit et sur les outils pour les détecter ou les éviter (Bruillard et Blondel, 2007).

Si les apports de l'analyse des usages sont de nous éclairer sur ce que les utilisateurs font ou non et avec quelles significations, les limites en sont qu'elle ne nous renseigne pas sur ce qu'ils pourraient faire. Les réflexions sur l'éducation cherchent à traiter cette seconde question. Pour ce faire, un point de vue historique peut être pertinent. Mais reste à définir selon quels modèles et pour cela d'avoir cerné, au moins en partie, l'informatique.

\section{Trois visions de l'informatique}

Il y a de nombreuses manières de reconstruire l'histoire de l'informatique. Si, comme nous venons de le voir, on prend le prisme des utilisateurs, des systèmes techniques et des applications, on constate une extension continue des premiers et une formidable diversification des deuxièmes et des troisièmes. Mais outre cette composante multiple liée aux usages, il y a également une composante liée à l'informatique elle-même, souvent en tension entre les TIC et la "science informatique " (ICT vs. computer science, Tort et Bruillard, 2010). A la fois une science et une technique, l'informatique n'est ni réductible aux ordinateurs, qui ne sont que des instruments de cette science, ni à la programmation, nécessaire mais qui se situe à un niveau uniquement microscopique (Beaudoin-Lafon, 2009). Selon Dowek (2010), «l'informatique est centrée autour de quatre concepts: le concept de machine, le concept d'algorithme, le concept d'information et le concept de langage ». Mais il ajoute que la notion d'information est propre à l'informatique, ce qui n'est sans doute pas un avis partagé par nombre de chercheurs en sciences de l'information et de la communication.

14 Bruillard (2010) caractérise l'informatique autour de trois approches complémentaires et souvent hybridées: (1) algorithmique et traitements automatisés autour du cycle données/traitement/résultats ; (2) interaction continue avec des machines, des artefacts sémiotiques, dans ce qu'Anne Nicolle (2003) nomme des processus à durée indéfinie ; 
(3) participation à des interactions sociales avec des agents humains et non humains via les réseaux. Cette tripartition permet de souligner les différents éléments concourant à une culture informatique qu'il reste à construire: une "pensée» informatique, la maîtrise d'objets informatiques et la participation à des activités sociales dans un monde en réseau.

\section{Démarche intellectuelle}

Plus que des savoirs et savoir-faire, des auteurs insistent sur la pensée informatique inhérente à la culture informatique depuis une quarantaine d'années. Baron et Bruillard (1996) proposent une chronologie de l'introduction de l'informatique en milieu scolaire. Dans les quatre phases identifiées, la deuxième (1970-1980) est caractérisée par l'idée de démarche informatique. Ainsi, Hebenstreit (1978) propose l'enseignement d'une démarche algorithmique. Cette idée de démarche de pensée informatique est reprise par d'autres chercheurs (Arsac, 1981; Breton, 1990; Mirabail, 1990). Arsac (1981) la caractérise « comme algorithmique, opérationnelle, organisationnelle ».

Plus récemment, Jeannette Wing (2006), définissant la pensée computationnelle, insiste sur le fait qu'« il s'agit bien d'une pensée, pas simplement de calcul mécanique au sens de routinier ", mettant en œuvre plusieurs niveaux d'abstraction. Elle met en exergue la résolution de problème, la capacité que procure cette forme de pensée particulière à formuler le problème, le décomposer, bref, le penser autrement pour le résoudre. C'est aussi selon elle la capacité à nommer les objets pour pouvoir les manipuler de manière abstraite. Il s'agirait de former l'esprit à une pensée singulière, utile dans d'autres sciences et dans la vie quotidienne. Mais cette pensée n'est pas pure abstraction car elle est ancrée dans une pensée technologique, du fait que les systèmes ainsi conçus interagissent avec le monde réel.

\section{Interactions avec les objets}

17 En effet, une autre dimension à prendre en compte est celle des objets informatisés, avec lesquels nous interagissons quotidiennement. Selon Norman (1987), les utilisateurs développent un modèle mental d'un système en interagissant avec lui. Cette construction s'opère à partir des utilisations faites et d'une image du système, qui donne ou devrait donner à voir toutes les informations nécessaires à son utilisation. Les propriétés perceptives, les "affordances" sont censées permettre des utilisations directes, immédiates, sans difficulté, du moment qu'une certaine " habitude » s'est installée. Que les utilisateurs en soient conscients ou non, leurs modes d'utilisation réfèrent à ce modèle mental. Bien évidement, les problèmes sont liés aux écarts possibles entre ce modèle mental et le modèle conceptuel sous-jacent au système ou à son modèle de fonctionnement.

Or, la construction s'opère au cours de processus interactifs de durée indéfinie, relevant de nombreuses applications: traitements de texte, tableurs, gestionnaires de bases de données ou de mails, butineurs Web (Nicolle, 2003). Dans ces processus, l'interprétation humaine est partie intégrante et la compréhension qu'ont les humains de ce que leur montre les machines est déterminante. Souvent, ce sont des interfaces dites de manipulation directe qui sont proposées, engageant directement les utilisateurs dans 
l'action. Les répétitions d'actions concourent à la mise en place de «gestes» (que l'on peut qualifier selon les théories de schèmes, d'habitus, de disposition...).

19 La relation entre les gestes appropriés et la conceptualisation, permettant une appréhension beaucoup plus profonde des systèmes, est au cœur d'une culture informatique à construire, assurant un lien entre les expériences des utilisateurs et les concepts sous-jacents aux systèmes utilisés et que l'utilisation seule ne permet de découvrir ou de construire. Le chaînon manquant est souvent la langue, c'est-à-dire la verbalisation des situations, permettant de prendre de la distance par rapport aux actions et de construire les concepts.

\section{Informatique sociale}

Depuis l'extension de l'Internet, il ne s'agit plus d'une interaction individuelle avec ou via un objet informatisé, selon des protocoles stabilisés. Ce ne sont plus seulement les interprétations des usagers et leurs choix qui orientent les traitements, mais également l'activité d'une multitude d'agents humains et non humains.

21 C'est sans doute en ce sens que Marchionini (2008) indique que beaucoup d'interactions personne-machine sont devenues des « interactions personne-information », par exemple dans le fonctionnement actuel du web. Il ne s'agit pas seulement d'interagir avec des objets mais aussi d'interagir avec les informations, produites par l'utilisateur lui-même ou par d'autres utilisateurs. Ces utilisateurs ne se contentent plus de consommer l'information (la lire, l'écouter, la regarder, etc.), mais interagissent avec elle. Les tags, les annotations, les commentaires en sont quelques manifestations. Ainsi, la navigation sur les sites, notamment de e-commerce et les moteurs de recherche, peuvent être historisées et servir à proposer des recommandations ou à adapter les produits, les publicités ou encore les résultats affichés. L'activité individuelle prend place dans un «flot » collectif. Les traces organisées des activités des autres, parfois de beaucoup d'autres, fournissent une énergie potentielle, qualifiée parfois d'intelligence collective, permettant de réduire la complexité algorithmique des outils informatiques (Lancieri, 2005): réduction optimisée d'espace d'information, économie de ressources, augmentation de la rapidité de traitement et de la qualité du résultat de certaines fonctions comme le tri (recherche d'informations).

Ce sont également des formes d'activités collectives qui sont facilités par les technologies de réseau et des changements dans les rapports au temps avec des incidences fortes dans de nombreuses activités humaines et sociales. L'informatique participe de ce mouvement mais donne des outils pour le suivre, parfois pour mieux le comprendre. Ce mouvement est analysé par Doueihi (2008) comme un véritable processus civilisateur. La culture numérique définirait des normes d'admission et d'appartenance dans les collectifs en ligne. Cette nouvelle civilité organiserait la présence et la représentation numérique de l'individu en fonction de la réalité technique, qui contraint les pratiques humaines. $\mathrm{Ce}$ processus bousculerait des normes et conventions établies, relevant de la culture de l'imprimé : propriété intellectuelle (droits d'auteur, fonction d'auteur, etc.), conception géographique et généalogique de l'identité par exemple.

Ainsi, la culture informatique aurait plusieurs dimensions. Mais la «pensée informatique " est souvent niée. On prétend que les interfaces actuelles ont fait disparaître toute difficulté d'interaction avec les machines et les personnes, surtout les jeunes, sont censées être acculturées au monde en réseau. 


\section{Conclusion} conscience qu'elles produisent des traces, que la capacité de stockage et de recherche d'information des objets informatisés en font une véritable mémoire. Ne pas laisser cette mémoire se constituer à l'insu de l'individu implique la maîtrise des objets informatisés en jeu. C'est comprendre que ces objets ne sont pas exogènes aux sociétés dans lesquelles ils trouvent place. C'est aussi être capable de "pensée informatique». Au final, ces cultures, informatique et du numérique, ont beaucoup à voir avec une culture informationnelle, qui mettrait au cœur l'information sans exclure l'informatique, dans une perspective critique et citoyenne. Reste à bâtir cette éducation aux médias, à l'information ou aux TIC.

Pour expliquer le choix et la légitimation des contenus d'enseignement, les modèles les plus utilisés sont celui de la transposition didactique et celui de la référence à des pratiques sociales. S'agissant d'informatique ou de TIC, ces deux modèles ne sont pas vraiment opératoires. Le premier évacue les cultures numériques et renforce la constitution d'un îlot disciplinaire. Le second suppose des pratiques stabilisées et reconnues qui puissent servir de référence. Faire converger l'éducation à l'information, aux TIC et aux médias invite à la mise en place d'autres processus de choix et de légitimation. Des constructions sont encore à penser, la convergence entre éducation à l'information, aux TIC et aux médias peut buter sur les cultures des enseignants susceptibles de se reconnaître dans ses différentes éducations. Trouver et former des enseignants partageant ces différentes cultures est loin d'être un problème réglé.

\section{BIBLIOGRAPHIE}

Arsac J., (1981), Informatique et enseignement général : http://www.epi.asso.fr/revue/histo/ h80simon-arsac.htm, consulté le 23 septembre 2011.

Arsac J., (1987), Les machines à penser. Des ordinateurs et des hommes, Paris, Seuil.

Agence wallonne des télécommunications, (2007), Citoyens wallons : usages TIC 2007, 115 p. : http://www.awt.be/contenu/tel/dem/usages_citoyens_2007.pdf, consulté le 23 sepmbre 2011.

Baron G.-L. \& Bruillard É., (1996), L'informatique et ses usagers dans l'éducation, L'Éducateur, Paris, Presses universitaires de France.

Baron G.-L. \& Bruillard É., (2008), Technologies de l'information et de la communication et indigènes numériques : quelle situation?, Rubrique STICEF, Volume 15, 2008 : http://sticef.univ-lemans.fr/num/ vol2008/09r-baron/sticef_2008_baron_09.htm.

Beaudoin-Lafon M., (2010), Informatique : information, interaction et automatisation... Actes du colloque, Didapro 3 : http://www.epi.asso.fr/revue/articles/a1005c.htm.

Études de communication, 38 | 2012 
Breton P., (1990), « La notion de 'culture informatique' : ambiguïté ou pluralité », in Breton P., Rieu A.-M. et Tinland F., La techno-science en question, «Éléments pour une archéologie du XX siècle », Champ Vallon, coll. « Milieux », Seyssel, 1990, (pp. 202-209).

Brown Polly S. \& Gould John D., (1987), An experimental study of people creating spreadsheets, ACM Transactions on Information Systems (TOIS), Volume 5, Issue 3, ACM Press, pp. 258-272.

Bruillard É., (2010), Acteurs et territoires de l'éducation à l'information : un point de vue " informatique ", in Chapron F. et Delamotte E. (dirs.), L'éducation à la culture informationnelle, Villeurbanne, Presses de l'ENSSIB, pp. 68-75.

Bruillard É. et Blondel F.-M., (2007), Histoire de la construction de l'objet tableur. pré-publication, hal-00180912, version 1, 32 p. : http://hal.archives-ouvertes.fr/hal-00180912/fr/.

Casili A., (2011), Cultures du numérique, Communications, $n^{\circ} 88$.

De Noblet J., (1982), La technique dans la culture, Esprit, octobre, Paris, pp. 8-16.

Demazière D., Horn F. \& Zune M., (2009), Les développeurs de logiciels libres : militants, bénévoles, ou professionnels? - Un regard socio-économique sur les communautés de développement de logiciels libres, in D. Demazière \& C. Gadéa (Éds.), Sociologie des groupes professionnels, Recherches, Paris, La Découverte.

Denouël J. et Granjon F., (2011), Communiquer à l'ère numérique. Regards croisés sur la sociologie des usages, Collection Sciences sociales, France, Paris, Transvalor-Presses des Mines.

Doueihi M., (2008), La grande conversion numérique, Paris, Éd. Du Seuil, La librairie du XXI ${ }^{\mathrm{e}}$ siècle.

Dowek G., (2010, septembre), Un chemin initiatique vers l'abstraction : http://www.epi.asso.fr/ revue/articles/a1009g.htm, consulté le 23 septembre 2011.

Dupagne D., (2011), E-santé, Communications, n 88, Cultures du numérique, pp. 57-65.

Fluckiger C., (2008), L'école à l'épreuve de la culture numérique des élèves, Revue française de pédagogie, (163), 51-61.

Fourmentraux J.-P., (2011), Net Art, Communications, n 88, Cultures du numérique, pp. 113-120.

Granjon F., (2011), Fracture numérique, Communications, n 88, Cultures du numérique, pp. 67-74.

Hebenstreit J., (1978), Nouvelles tendances en enseignement assisté par ordinateur : http:// upcommons.upc.edu/revistes/bitstream/2099/4596/4/article.pdf, consulté le 23 septembre 2011.

Jouët J., (1990), L'informatique « sans le savoir », Culture technique, (21), 216-222.

Jouët J., (2011), Des usages de la télématique aux Internet Studies, in J. Denouël \& F. Granjon (Éds.), Communiquer à l'ère numérique. Regards croisés sur la sociologie des usages, Collection Sciences sociales (pp. 45-90), France, Paris, Transvalor-Presses des Mines.

Lancieri L., (2005), Interactions humaines dans les réseaux, Cachan, Hermès - Lavoisier, 208 p.

Lobet-Maris C., (2011), Âge et usages informatiques, Communications, $n^{\circ} 88$, Cultures du numérique, pp 19-28.

Marchionini G., (2008), Human-information interaction research and development, Library \& Information Science Research, 30(3), 165-174.

Mirabail M., (1990), La culture informatique, Aster, (11), pp. 11-28.

Norman D. A., (1987), Some observations on mental models, in R. M. Baecker (Éd.), Human-computer interaction (pp. 241-244), San Francisco, CA, USA, Morgan Kaufmann Publishers Inc. 
Nicolle A., (2003), Étude préliminaire à une théorie des processus interactifs infinis, Cahiers du Greyc| UMR6072 - GREYC - Groupe de REcherche en Informatique, Image, Automatique et Instrumentation de Caen.

Panko R., (2000), What We Know About Spreadsheet Errors : http://panko.shidler.hawaii.edu/SSR/ Mypapers/WhatKnow.htm, consulté le 23 septembre 2011.

Perriault J., (1990), L'empreinte de l'ordinateur sur les modes de pensée des utilisateurs, Culture technique, (21), 236-245.

Proulx S., (1990), La promotion sociale de la « culture informatique ». Du « computer power to the people » à l'efficacité d'un nouvel outil pour le travail de bureau, Culture technique, (21), pp. 223-235.

Tort F. \& Bruillard É., (2010), Informatics education : beyond the opposition between information technology and computer science, in D. Benzie, K.-W. Lai \& C. Reffay (Éds.), New Developments in ICT and Education, proceedings of IFIP Working Conference, ISBN : 978-2-9537285-1-4.

Turkle S., (1984), The Second Self: Computers and the Human Spirit, New York, Simon \& Shuster.

Voirol O., (2011), L'intersubjectivation technique : de l'usage à l'adresse. Pour une théorie critique de la culture numérique, in J. Denouël \& F. Granjon (Éds.), Communiquer à l'ère numérique. Regards croisés sur la sociologie des usages, Collection Sciences sociales (pp. 127-157), Paris, Paris, Transvalor-Presses des Mines.

Wing J., (2006), Computational Thinking, Communications of the ACM, March 2006/Vol. 49, n³, 33, traduction française : http://interstices.info/jcms/c_43267/la-pensee-informatique, consultée le 23 septembre 2011.

\section{RÉSUMÉS}

L'éducation aux technologies de l'information et de la communication (TIC) devrait tenir compte des cultures communes et partagées, ou supposées telles, par les élèves. La définir aujourd'hui demande aussi de mieux cerner l'« objet » informatique, dans une perspective historique, par les cultures qui lui sont associées, et dans la manière de penser cet objet. En effet, les évolutions qu'a connues l'informatique pourraient laisser penser que nous sommes passés, en une trentaine d'années, des cultures informatiques aux cultures numériques. Les premières seraient focalisées sur le code et la connaissance des langages de programmation. Les secondes seraient centrées sur les usages, grâce à des interfaces toujours plus conviviales et transparentes. Or ces deux types de cultures coexistent aujourd'hui sans que l'on puisse considérer qu'il y ait eu évolution de l'une vers l'autre. Les discours sur ces cultures renvoient en fait à une conception de l'informatique qu'il convient de mettre en perspective pour pouvoir réfléchir sur la thématique de l'« éducation à » concernant les TIC. Nous exposons dans cet article trois visions de l'informatique qui nous semblent particulièrement significatives pour éclairer ce débat : une démarche intellectuelle, des interactions avec les objets et une informatique sociale, basée sur la coopération entre acteurs et agents et l'intelligence collective.

Educational approaches to information and communication technology (ICT) should take into account the cultures shared (or thought to be shared) by students. Defining such education today also requires that the technical focus be more fully understood not only from a historical perspective but also with respect to the cultures associated with it and the ways in which it is conceived. Developments in computer science might suggest that within thirty years, we have moved from computer fluency to a digital culture. While the former focuses on codes and on knowledge of programming languages, the latter is centered on usage, thanks to increasingly 
intelligible and user-friendly interfaces. Yet these two types of culture currently exist side by side without any shift of the one toward the other. A discussion of these cultures reveals a particular conception of computer science analyzed here in terms of the question of education with regard to ICT. We then set out three views of information technology that seem particularly meaningful in clarifying the debate: an intellectual approach, interaction with objects, and a social informatics based on cooperation among actors or agents (humans and nonhumans) and collective intelligence.

\section{INDEX}

Keywords : computational thinking, teaching methods, usage, technical culture, information literacy

Mots-clés : pensée informatique, didactique, usages, culture technique, culture informationnelle

\section{AUTEURS}

\section{BÉATRICE DROT-DELANGE}

Laboratoire ACTé - Université Blaise Pascal Clermont-Ferrand

ÉRIC BRUILLARD

Laboratoire STEF - ENS Cachan 